\title{
Energieautarke automatische EKG-Erfassung mit drahtloser Datenübertragung für die Langzeitdiagnose
}

Klaus Dembowski, Tom Trockels, Hoc Khiem Trieu

Technische Universität Hamburg-Harburg, Institut für Mikrosystemtechnik

Eissendorferstr. 42, 21073 Hamburg

040/42878-3229, trieu@tu-harburg.de, dembowski@tu-harburg.de

Keywords: EKG-Messsystem, drahtlos, energieautark, Energy Harvesting, Energy Management

\section{Einführung}

Im medizinischen Bereich sind die Vitalparameter eines Menschen von großer Bedeutung, die die Grundfunktionen des menschlichen Körpers widerspiegeln und sowohl diskrete Messungen

(Körpertemperatur, Herzfrequenz, Blutdruck, Atmung) als auch kontinuierliche wie das

Elektrokardiogramm (EKG) und das Elektroenzephalogramm (EEG) umfassen. Vitalparameter werden traditionell im klinischen und zunehmend im häuslichen Umfeld erfasst. Gerade hier ist ein großer Bedarf an wartungsfreien energieautarken Patientensystemen (home care, wellness) zu verzeichnen, die eine kontinuierliche Aufzeichnung von Vital- und Gesundheitswerten gestatten und für genauere Auswertungen bereitgestellt werden können.

Die lokale Datenspeicherung der Parameter erfolgt oftmals auf einer SD-Card (SecureDigital) und ähnlichen Speicherkarten, die aus dem Mobilsystem entfernt und für die Auswertung typischerweise in eine PC-gestütztes Lesegerät einzusetzen sind. Alternativ und weniger aufwendig in der Handhabung ist eine Datenübertragung per Standardschnittstelle wie dem Universal Serial Bus (USB) zwischen der mobilen Einheit und dem Auswertungssystem, was jedoch ebenfalls manuelle Handreichungen wie dem Ein- und Abstecken des Datenkabels notwendig macht und stets als potentielle Fehlerquelle anzusehen ist.

Die Datenübertragung per Funk macht Kabelverbindungen überflüssig, wenn die mobile Einheit energieautark gestaltet wird, also keine Kabelverbindungen für die Spannungsversorgung benötigt werden. Energieautarkie wird prinzipiell bereits durch Batterien erreicht, deren Lebensdauer meist geringer ist als die des Gerätes, welches sich andernfalls nicht amortisieren würde, so dass ein Austausch der Batterien notwendig wird. Der Einsatz von Akkumulatoren verlängert zwar den Zeitpunkt des notwendigen Austausches der Spannungsquelle oder macht ihn sogar überflüssig, es setzt jedoch ein regelmäßiges Aufladen der Zellen voraus, wofür wieder eine Kabelverbindung herzustellen und eine regelmäßige Ladezustandsprüfung des Akkus notwendig ist.

Für wartungsfreie energieautarke Systeme, bei denen weder Primär- noch Sekundärzellen zur Anwendung kommen sollen, die sich durch eine einfache Handhabung auszeichnen und die die Messdaten drahtlos für die Weiterverarbeitung an eine Basisstation übertragen können, ist deshalb eine autarke lokale Form der Energiegewinnung notwendig. Das realisierte EKG-System basiert deshalb auf dem Prinzip des Energy Harvesting [1] und erzeugt die für den Betrieb notwendige Energie allein aus der Umgebung mithilfe einer Indoor-Solarzelle.

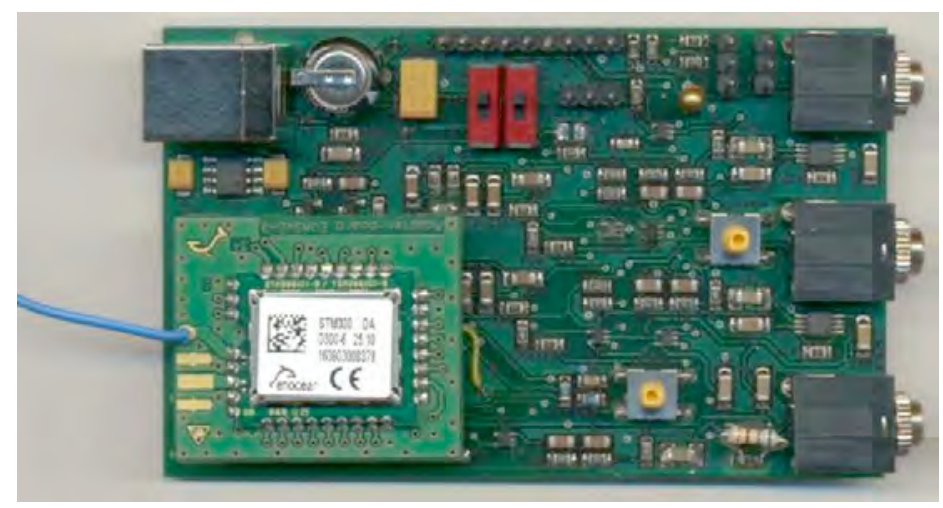

Abbildung 1: Die Platine des EKG-Messsystems

Die entwickelte Schaltung befindet sich die meiste Zeit in einem Sleep Modus und kann durch verschiedene Ereignisse manuell (patientengesteuert) oder automatisch (ereignisgesteuert) in ihren 
aktiven Zustand versetzt werden, was zu einer EKG-Aufzeichnung führt. Für ein EKG werden 300 Messwerte bei einer Abtastfrequenz von $160 \mathrm{~Hz}$ mit einer Auflösung von 10 Bit aufgenommen, was somit ein komplettes EKG-Signal liefert und genaue Analysen ermöglicht. Die über die Solarzelle gewonnene Energie wird in einem Doppelschichtkondensator (Goldcap) gespeichert und mithilfe eines EnergieManagements so verwaltet, dass nach mindestens 10 Minuten ausreichend Energie zur Verfügung steht, damit eine EKG-Aufnahme stattfinden kann. Ein voll aufgeladener Kondensator erlaubt acht Messungen/Übertragungen, ohne dass die Solarzelle Energie liefern muss. Für die Übertragung wird eine einfache Funktechnik bei $868 \mathrm{MHz}$ eingesetzt, die mit dem Modulationsverfahren Amplitude Shift Keying (ASK) arbeitet. Ein derartiges drahtloses und am Körper tragbares EKG-System ermöglicht die volle Bewegungsfreiheit eines Probanden und ist deshalb insbesondere für Langzeitmessungen prädestiniert.

Die Programmierung und Kalibrierung des Systems erfolgt über den USB eines an die Platine angeschlossenen Personal Computers, der einerseits die Entwicklungsumgebung bereitstellt und andererseits die Messsignale mithilfe eines Funktransceivermoduls empfangen und dementsprechend weiterverarbeiten (Speicherung, Visualisierung, Analyse) kann.

\section{Elektrokardiographie}

Die Elektrokardiographie hat sich seit langem als ein weit verbreitetes Verfahren zur Diagnose von Herzaktivitäten etabliert. Dabei werden elektrische Potenziale, die durch die Aktivität des Herzens entstehen und bis auf die Körperoberfläche gelangen, mithilfe von Elektroden gemessen. Deren Beschaffenheit und Position ist für verschiedene Methoden und Einsatzgebiete definiert. Für ein vollständiges EKG werden insgesamt 12 Kanäle vorausgesetzt, was genaue Analysen, etwa bei der Infarktdiagnostik in der Klinik ermöglicht, während für die Vitalparameterkontrolle, wie in der Notfallmedizin oder auch für Langzeit-EKGs, einfachere Methoden üblich sind, die mit lediglich drei Kanälen auskommen.

Die verschiedenen Abläufe eines Herzschlages werden durch elektrische Impulse gesteuert, die von dem so genannten Sinusknoten gesendet werden. Der Sinusknoten ist eine Ansammlung von Nervenzellen, die den Taktgeber des Herzens darstellt und in regelmäßigen Abständen eine elektrische Erregung erzeugt, die sich über das gesamte Herz ausbreitet. Die Überlagerung der Aktionspotentiale, die aus verschiedenen Herzregionen entstanden sind, bildet das EKG-Signal.

Die Änderungen der Aktionspotentiale am Herzen bzw. die EKG-Signale selbst lassen sich an der Körperoberfläche durch verschiedene Methoden ableiten. Diese EKG-Ableitungen haben unterschiedliche Anordnungen der Elektroden zur Folge, wofür verschiedene Signalberechnungsverfahren existieren. Die einzelnen Ableitungen erlauben eine Beurteilung von den in Richtung der Ableitung liegenden Herzabschnitten und registrieren den Verlauf der Potenziale aus verschiedenen Perspektiven. Die üblichen standardisierten Ableitungsmethoden in der Elektrokardiographie [2] sind die unipolare Ableitung nach Goldberger, die bipolare Ableitung nach Einthoven (I, II und III), und die Ableitung nach Wilson, die sechs zusätzliche Elektroden benötigt, die an der Brustwand zu positionieren sind. Für die Methoden nach Goldberger und Einthoven sind die Elektroden an den Extremitäten zu positionieren. Das entwickelte EKG-Messsystem ermöglicht eine Differenzmessung auf drei Kanälen, sodass hiermit Aufnahmen nach Einthoven (Abbildung 2) und auch Goldberger möglich sind. 


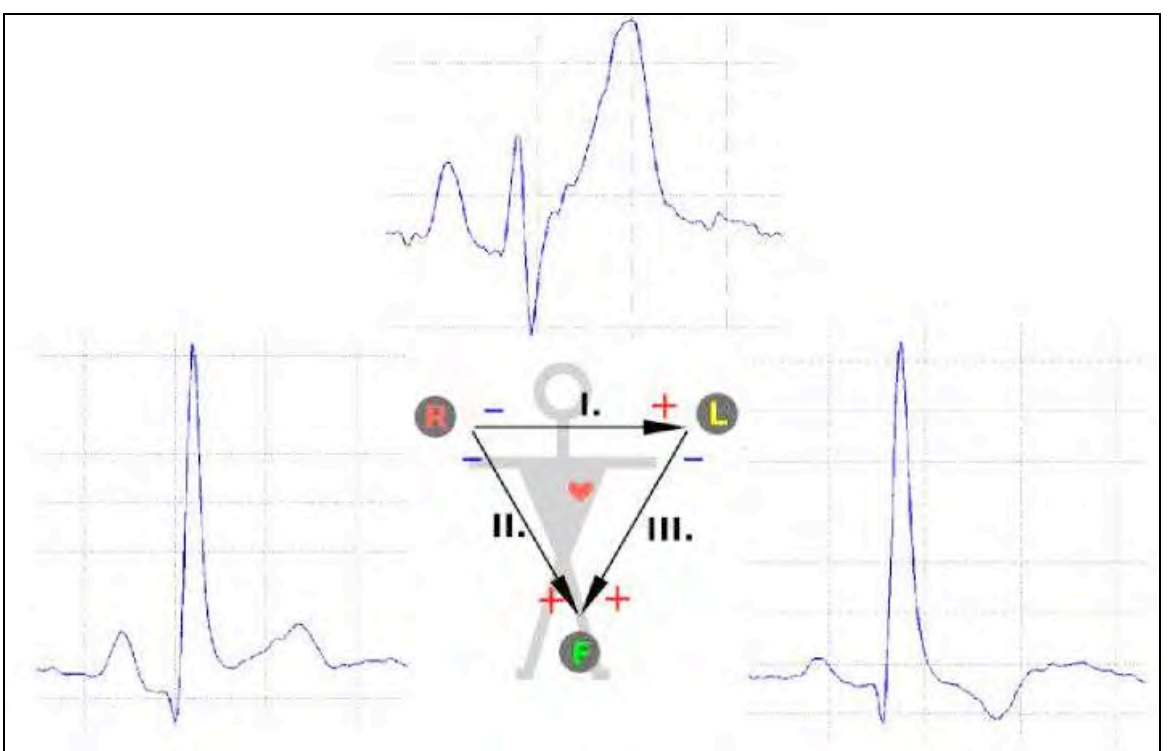

Abbildung 2: Positionen der Elektroden und die Signale entsprechend der Methode nach Einthoven [6]

Das System wurde nach Einthoven mit handelsüblichen Silber/Silberchlorid-Haftelektroden $(\mathrm{Ag} / \mathrm{AgCl})$ betrieben, die einen verhältnismäßig geringen Kontaktwiderstand aufweisen und zu den rauschärmsten EKG-Elektroden gehören. Der eingesetzte Typ Blue Sensor L von Ambu [3] ist explizit für

Langzeitüberwachungen vorgesehen und kann deshalb länger als andere ähnliche Elektroden auf der Haut verbleiben. Die Elektroden sind als so genannte EKG-Pads ausgeführt, die paarweise auf die Haut geklebt werden.

\section{Analoge Signalverarbeitung - EKG-Messverstärker}

Die EKG-Signale werden über ein Filter- und Verstärkungssystem (Messverstärker) verarbeitet, welches unerwünschte Gleichspannungs- und Hochfrequenzanteile filtert, die allgegenwärtigen $50 \mathrm{~Hz}$-Störungen wirkungsvoll unterdrückt und die Amplituden optimal an den Messbereich des nachgeschalteten A/DWandlers anpasst. Um eine medizinisch auswertbare EKG-Messung zu erhalten, wird die zeitgleiche Aufzeichnung aller drei Ableitungen nach Einthoven benötigt. Die Schaltung besteht deshalb aus drei Messverstärkerzweigen für die EKG-Signale sowie einer Temperaturmessschaltung, die für übliche Thermistoren (NTC) geeignet ist, um hiermit eine optionale Messung der Körpertemperatur zu ermöglichen, die aber nicht Gegenstand der weiteren Betrachtungen ist.

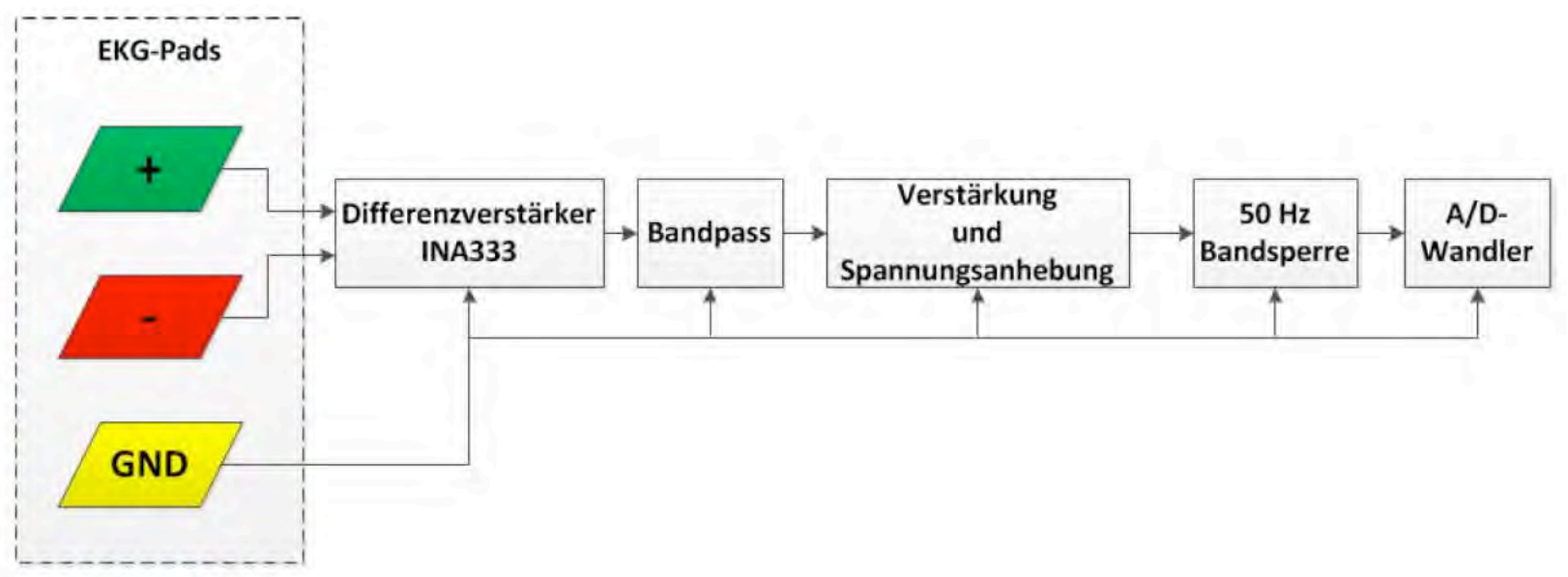

\section{Abbildung 3: Die Signalaufbereitungsschaltung für EKG-Signale}

Das eingespeiste differentielle EKG-Signal der Elektroden gelangt auf einen Differenzverstärker (INA333) der Firma Texas Instruments, der durch einen maximalen Offset von $25 \mu \mathrm{V}$, einer temperaturabhängigen Drift von $0,1 \mu \mathrm{V} /{ }^{\circ} \mathrm{C}$ sowie durch einen Stromverbrauch von $50 \mu \mathrm{A}$ spezifiziert ist. Der „Innenwiderstand des menschlichen Körpers" und die dazu in Reihe liegenden Übergangswiderstände der Elektroden bilden den $R_{k}$-Innenwiderstand der Signalquelle. Das EKG-Signal $\left(U_{e k g}\right)$ und der $R_{k}$-Innenwiderstand führen zu in einer vereinfachten Darstellung des Ersatzschaltbildes (Abbildung 5) für die EKG-Messung. 


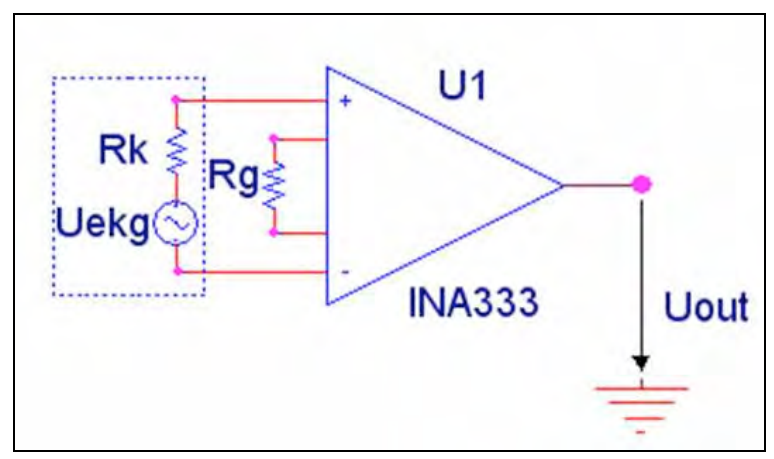

Abbildung 4: Schaltbild der EKG-Eingangsstufe

Der Rk-Innenwiderstand liegt im k $\Omega$-Bereich und ist im Vergleich zur Eingangsimpedanz des INA333Differenzverstärkers, die im $G \Omega$-Bereich liegt, vernachlässigbar klein. Aufgrund der sehr hochohmigen Eingangsimpedanz des Differenzverstärkers wird das Messsignal kaum belastet. Die Verstärkung, die im Bereich von 1 bis 1000 liegen kann, wird mit dem Widerstand Rg, der zwischen Pin 1 und Pin 8 angeschlossen ist, bestimmt. Für Rg wurde ein Widerstand von $400 \Omega$ verwendet, womit sich die Verstärkung zu einem Wert von ca. 250 ergibt.

$$
G=1+\left(\frac{100 k \Omega}{R_{g}}\right)
$$

Das Ausgangssignal des Differenzverstärkers wird in der zweiten Stufe durch ein Hochpassfilter 1. Ordnung verarbeitet. Dieser ist insbesondere für eine Abschwächung von Gleichspannungsanteilen mit $20 \mathrm{~dB} /$ Dekade vorgesehen, wofür ein einfaches RC-Glied zum Einsatz kommt. In Kombination mit dem darauf folgenden aktiven Tiefpassfilter 2. Ordnung (Sallen\&Key), der für das Unterdrücken der höheren Frequenzanteile im EKG-Signales bestimmt ist, ergibt sich ein Bandpassverhalten mit einer oberen Grenzfrequenz bei $70 \mathrm{~Hz}$ und einer Dämpfung von 20 dB/Dekade.

In der darauf folgenden Verstärkungsstufe, die ebenfalls mit einem Operationsverstärker vom Typ AD 8500 der Firma Analog Devices aufgebaut ist, wird das Messsignal verstärkt und im Pegel so verschoben, dass der Aussteuerbereich des A/D-Wandlers (Single Ended, 0-2 V) ausgenutzt werden kann. Diese Verstärker-/Anpassungsstufe ist als Differenzverstärkerschaltung (Subtrahierer) ausgeführt. Durch das Anlegen einer definierten Spannung (+Ub) an den nichtinvertierten Eingang des Operationsverstärkers wird das bipolare Messsignal mit einer Gleichspannung überlagert. Diese Gleichspannung soll der mittleren Spannung des Messbereiches entsprechen. Die Verstärkung ist mit den Widerständen R1 und R2 auf den Faktor 10 festgelegt. Mit Hilfe des Maschenstromverfahrens und der Annahme, dass es sich hier um einen idealen Operationsverstärker handelt, gilt die Gleichung:

$$
U_{\text {out }}=U_{b} \cdot\left(\frac{R_{1}+R_{2}}{R_{1}}\right) \cdot\left(\frac{R_{4}}{R_{3}+R_{4}}\right)-U_{\text {in }} \cdot \frac{R_{2}}{R_{1}}
$$

Das Messsignal $\bigcup_{\text {in }}$ erscheint mit einem negativen Vorzeichen in der obigen Gleichung. Weil das Messsignal mit dem Differenzverstärker zuvor invertiert wurde, ist es am Ausgang der zweiten Verstärkerstufe wieder mit dem richtigen Vorzeichen zu messen. $U_{b}$ hat einen festen Wert von $1,8 \mathrm{~V}$. Zusammen mit den Widerstandverhältnissen kann die mittlere Spannung des Messbereiches (900 mV), auf die das Messsignal angehoben werden soll, eingestellt werden. Mit der Bedingung $U_{\text {in }}=0 \mathrm{~V}$, eingesetzt in die obige Gleichung, kann die mittlere Spannung in Abhängigkeit von den Widerständen und der Spannung $U_{b}$ berechnet werden. Wenn die Hälfte des Messbereiches Ub/2 und die Verstärkung Faktor 10 entsprechen soll, ergibt sich mit $U_{\text {in }}=0 \mathrm{~V}$ die folgende Beziehung:

$$
\frac{1}{2}=10 \cdot\left(\frac{R_{4}}{R_{3}+R_{4}}\right) \quad \rightarrow \quad R_{3}=21 \cdot R_{4}
$$

Damit wird eine Dimensionierung mit $\mathrm{R} 1=100 \mathrm{k} \Omega, \mathrm{R} 2$ und $\mathrm{R} 3=1 \mathrm{M} \Omega$ sowie $\mathrm{R} 4=47 \mathrm{k} \Omega$ realisiert. Der erläuterte Bandpass ermöglicht die Übertragung von Frequenzen im Bereich von Gleichspannung bis zu $70 \mathrm{~Hz}$, was demnach keine $50 \mathrm{~Hz}$-Unterdrückung beinhaltet. Für eine effektive Unterdrückung der Netzfrequenz, die sehr stark in die Messung einstreuen kann, ist deshalb als letzte Stufe der 
Signalverarbeitungskette eine Bandsperre (Notch-Filter) notwendig. Am Markt sind zwar verschiedene Typen mit einstellbarer Resonanzfrequenz und Güte verfügbar, die aber nicht mit einer Betriebsspannung von 1,8 V auskommen und einen Stromverbrauch im $\mathrm{mA}$-Bereich aufweisen, was sich deshalb für eine energieeffiziente Lösung nicht eignet. Eine weitere Möglichkeit wäre ein Resonanzfilter, welches aus einer RLC-Schaltung besteht. Diese Lösung verlangt bei niedrigen Frequenzen jedoch eine verhältnismäßig große Induktivität oder die Verwendung von Ersatzschaltungen, was einer effizienten Lösung ebenfalls entgegensteht. Deshalb wurde eine passive Doppel-T-Schaltung als Filter realisiert.

Der RCR-Zweig (Abbildung 5) stellt ein Tiefpassfilter dar, und der CRC-Zweig ein Hochpassfilter. Die Parallelschaltung der beiden Filterschaltungen ergibt damit eine Bandsperre. Die beiden Übertragungsfunktionen schneiden sich genau bei der Sperrfrequenz, bei der die Übertragungsfunktion des Doppel-T-Filters gleichzeitig das Minimum erreicht. Die Übertragungsfunktion des Doppel-T-Filters kann mit Hilfe des Knotenpotentialverfahrens berechnet werden.

$$
H(j \omega)=\frac{U_{o u t}(j \omega)}{U_{\text {in }}(j \omega)}=\frac{1-\omega^{2} R^{2} C^{2}}{1+4 j \omega R C-\omega^{2} R^{2} C^{2}}
$$

Praktischerweise werden für alle Widerstände und Kondensatoren identische Werte vorgesehen. Wenn der Nenner der Gleichung auf Null gesetzt wird, d.h. es findet keine Übertragung statt, kann die Beziehung zwischen der Sperrfrequenz und den Bauteilgrößen der Filterschaltung berechnet werden. Die Bauelemente werden dann einfach berechnet mit:

$$
f_{\text {sperr }}=\frac{1}{2 \pi R C}
$$

Aufgrund der Tatsache, dass die Bauelemente entsprechend ihrer Baureihen nur mit bestimmten Standardwerten erhältlich sind, die auch gewisse Toleranzen aufweisen, ist zur genauen Anpassung mindestens ein Widerstandswert $\left(R^{*}\right)$ experimentell zu ermitteln, um somit eine exakte Dämpfung der Sperrfrequenz zu erreichen. Die Kondensatoren ergeben sich zu $33 \mathrm{nF}$ und die Widerstände zu jeweils $100 \mathrm{k} \Omega$, wobei für $\mathrm{R}^{*}$ ein Wert von $82 \mathrm{k} \Omega$ ermittelt wurde.

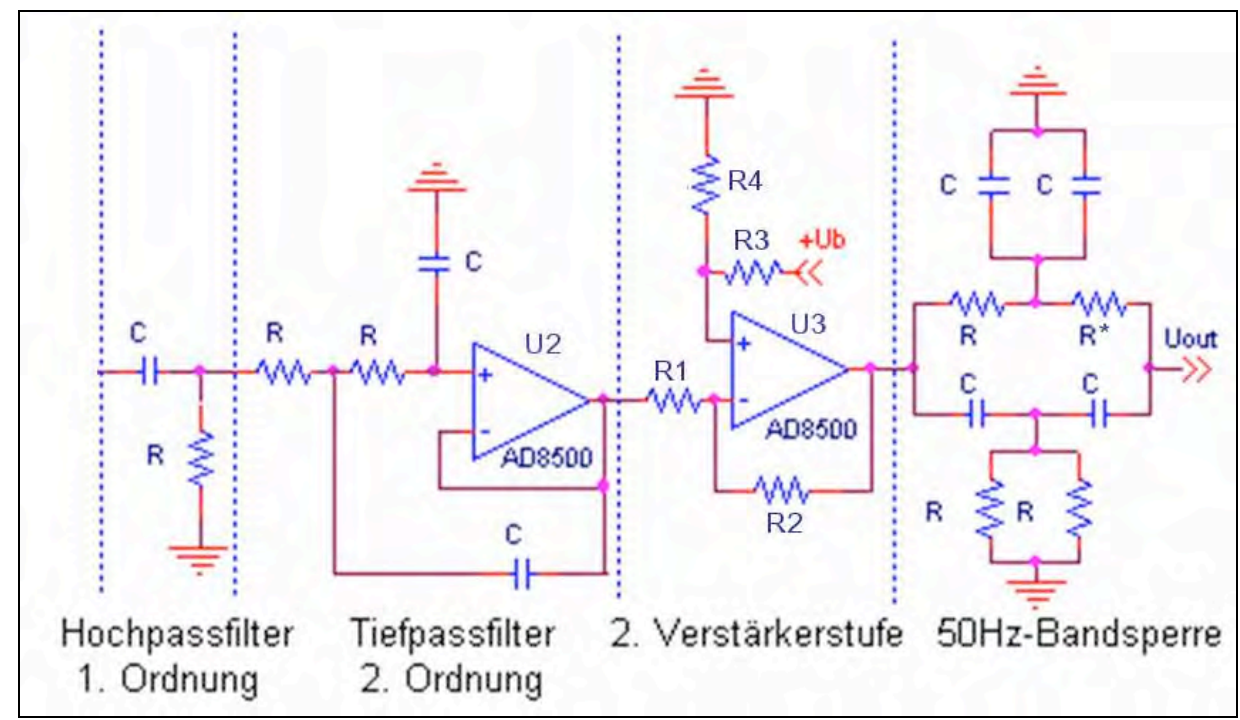

Abbildung 5: Schaltbild der Filter und der Verstärkerstufe mit Pegelanpassung (+Ub)

Die Übertragungsfunktion der gesamten EKG-Signalaufbereitungsschaltung ist in der Abbildung 6 gezeigt. Wie es zu erkennen ist, wird das Messsignal im Durchlassbereich mit 60 dB verstärkt. Durch das Hochpassfilter werden die Signalanteile für tiefere Frequenzen mit $20 \mathrm{~dB}$ zunehmend abgeschwächt. Die Auswirkung des Tiefpassfilters ist für Frequenzen ab $60 \mathrm{~Hz}$ zu erkennen. Da es sich um ein Tiefpassfilter 2. Ordnung handelt, fällt die Kurve mit einer Steigung von $40 \mathrm{~dB} /$ Dekade ab. Die Frequenzanteile bei 50 $\mathrm{Hz}$ werden im Vergleich zum Durchlassbereich mit $-40 \mathrm{~dB}$ abgeschwächt. 


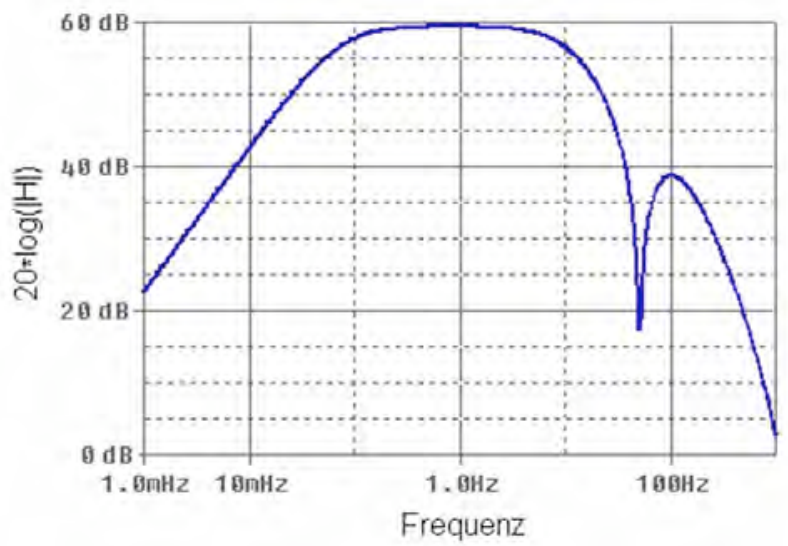

Abbildung 6: Übertragungsfunktion der Gesamtschaltung

Die Ausgangssignale $\left(U_{\text {out }}\right)$ der analogen Signalverarbeitung werden direkt auf die A/D-

Umsetzereingänge des Controllers STM 300 [4] geführt. Dieser enthält einen 8051-kompatiblen

Rechenkern mit Erweiterungen für ein Energie-Management sowie einen Transceiver für $868 \mathrm{MHz}$. Der STM 300 der Firma EnOcean enthält somit alle wesentlichen Einheiten, um die EKG-Applikation mit einer Steuereinheit (Microcontroller) und einem Funkinterface unter Einbeziehung von Energiesparmechanismen auszustatten.

Die Messverstärkerschaltung benötigt eine positive (+Ub) sowie die dazu äquivalente negative Spannung (Ub), was mithilfe eines Charge Pump/DC-DC-Konverters TC7660 von Microchip Technology erreicht wird. Als Spannungsquelle dient dabei der DVDD-Spannungsausgang des STM 300.

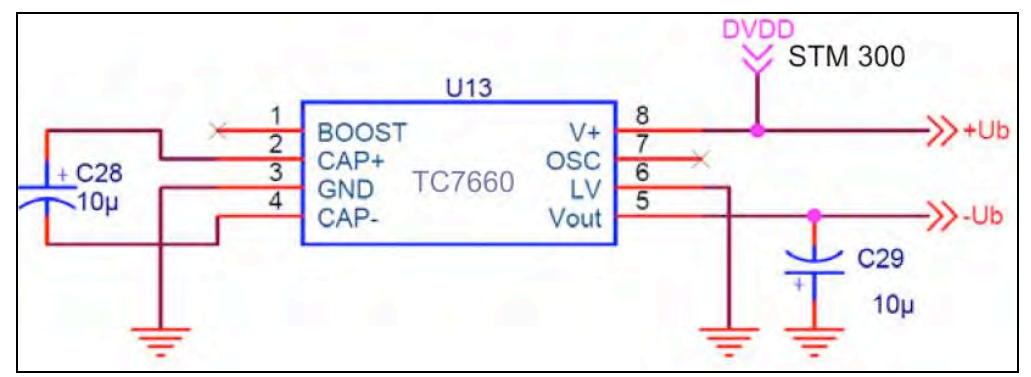

Abbildung 7: Die Spannungsversorgung für die Signalaufbereitung

\section{Störeinkopplungen per Software eliminieren}

Da die $50 \mathrm{~Hz}$-Bandsperre verhältnismäßig recht aufwendig ist, für eine optimale Funktion ein Ausmessen von Bauteilen $\left(R^{*}\right)$ erfordert und Energie verbraucht, hängt es von der gewünschten Signalqualität $a b$, ob nicht auf diese Stufe verzichtet werden kann und stattdessen eine entsprechende Signalnachbearbeitung auf einem PC, wo die EKG-Aufnahmen ohnehin weiterverarbeitet werden können, durchführbar ist.

Obwohl die Störeinkopplung durch das Stromversorgungsnetz bei $50 \mathrm{~Hz}$ oft sehr hoch ist, wirkt sie nur auf eine schmal begrenzte Bandbreite. Daher ist die Nachbearbeitung etwa mit Matlab von Vorteil, weil sie so präzise eingestellt werden kann, dass eine Beeinflussung des zu messenden EKG-Signals durch das Software-Filter unwahrscheinlich ist. Die Implementierung des Filters wird in Matlab mittels der diskreten Fast-Fourier Transformation (FFT), die die Messwerte vom Zeit- in den Frequenzbereich umsetzt, realisiert, so dass der Frequenzbereich daraufhin exakt gefiltert und anschließend wieder und in den Zeitbereich transformiert (inverse FFT) werden kann. In der Abbildung 8 sind ein ohne Bandsperre (in Hardware) aufgezeichnetes EKG-Signal sowie das durch Matlab gefilterte Signal gezeigt, bei dem das $50 \mathrm{~Hz}$-Rauschen vollständig eliminiert ist. 

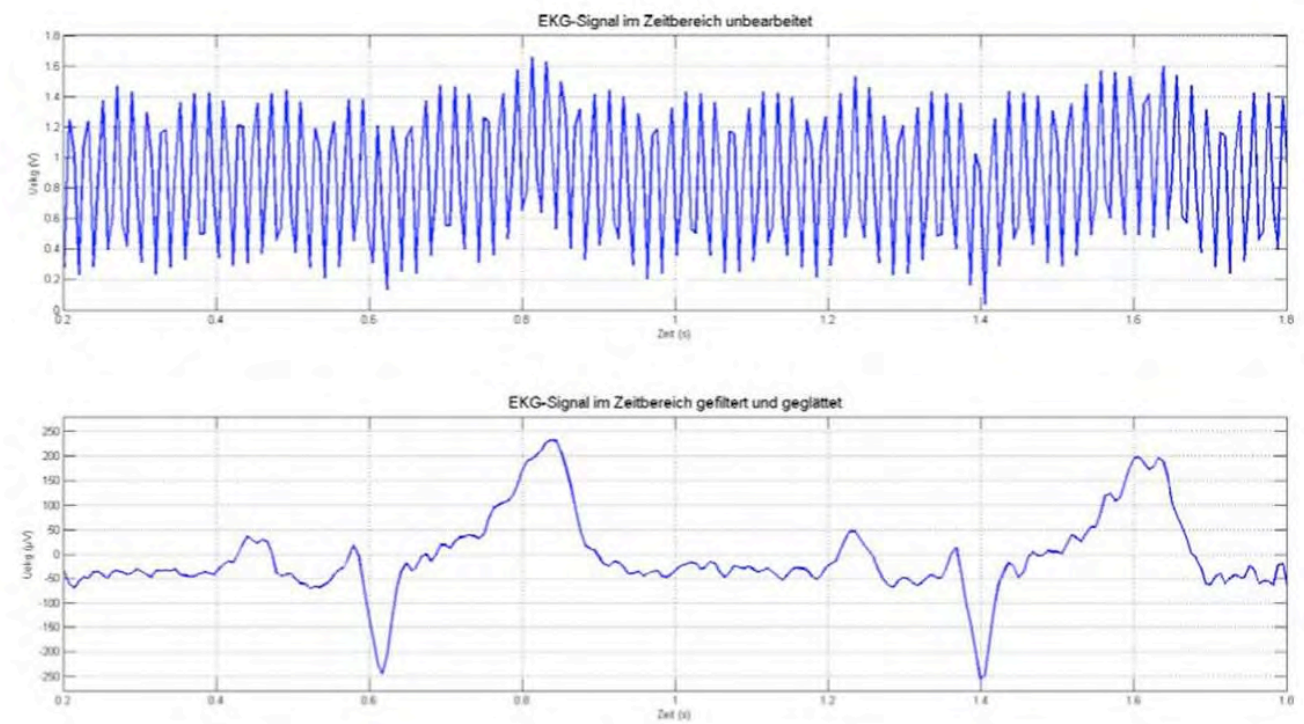

Abbildung 8: EKG-Signal ohne Bandsperre (oben), welches mithilfe von Matlab vom 50 Hz-Rauschen befreit wurde (unten).

\section{Scavenger Transceiver Module}

STM steht für Scavenger Transceiver Modul, welches für energieeffiziente auf Energy Harvesting basierte Applikationen, die eine Funkschnittselle benötigen, vorgesehen ist. Das Modul basiert auf einer mit $16 \mathrm{MHz}$ getakteten 8051-kompatiblen CPU mit 32 kByte Flash- und 2 kByte SRAM-Speicher. Integriert sind außerdem ein dreikanaliger A/D-Wandler (10 Bit), ein 8-Bit-D/A-Wandler, vier dezidierte I/O-Ports sowie drei Timer und es sind mehrere Anschlüsse für verschiedene Wake-Up- und Sende-Szenarien herausgeführt. Der Transceiver arbeitet mit einem speziellen, einfachen Protokoll von EnOcean mit einer ASK-Modulation bei $868,3 \mathrm{MHz}$ und ereicht eine Datenrate von $125 \mathrm{kBit} / \mathrm{s}$. Besonders verbreitet ist diese Funktechnologie in der Gebäudetechnik (drahtlose Schalter), die sich aufgrund ihrer Effizienz aber ganz allgemein für stromsparende Applikationen eignet.
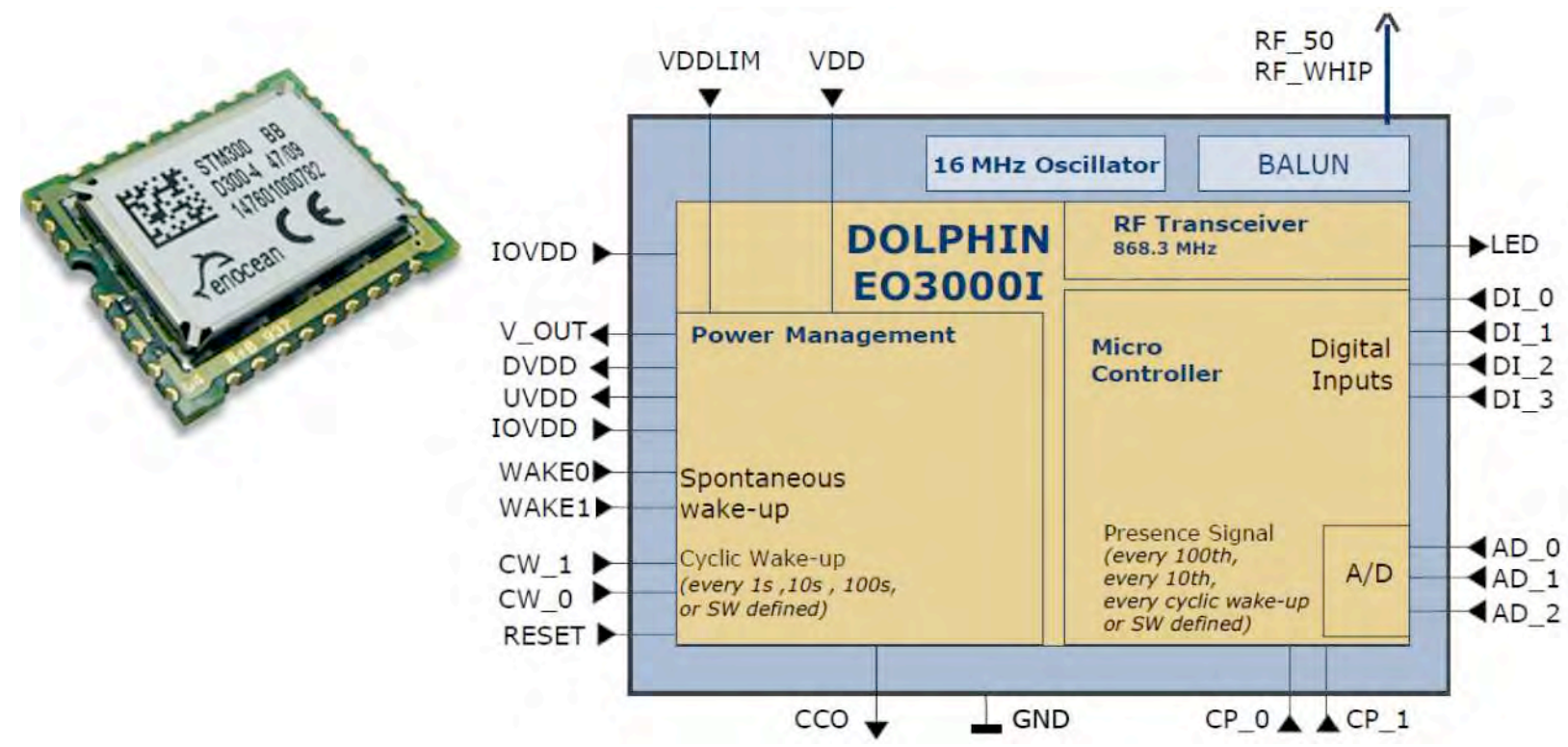

Abbildung 9: Das verwendete Scavenger Transceiver Modul der Firma EnOcean

Von besonderer Bedeutung für den Einsatz in Energy Harvesting-Anwendungen sind die verschiedenen Spannungs- und Power Management-Anschlüsse des Moduls. Die Versorgungsspannung (VDD) darf im Bereich von 2,1 V bis 4,5 V liegen, wobei für den Wake-Up aus einem Sleep-Modus jedoch mindestens 2,6 $\checkmark$ notwendig sind. Über den Anschluss VDDLIM (Voltage Limiter Input) wird ein Maximalwert für das Modul festgelegt, um etwa zu verhindern, dass ein angeschlossener Harvester das Modul mit einer zu hohen Spannung versorgen kann und es damit zerstört wird. 
Für die Spannungsversorgung externer Einheiten - wie der EKG-Schaltung - gibt es die Ausgänge V_OUT $(1,8 \mathrm{~V}, 10 \mathrm{~mA})$ und DVDD $(1,8 \mathrm{~V}, 5 \mathrm{~mA})$, die im Deep Sleep Mode abgeschaltet sind. UVDD (Ultra Low Power Supply) ist für den Einsatz mit den Wake-Up-Pins vorgesehen und darf nicht anderweitig angeschlossen werden. IOVDD ist ein Eingang für eine separate Spannungsversorgung (z.B.

Akkumulator), die nicht größer sein darf als VDD und mit DVDD verbunden wird, wenn keine zusätzliche Spannungsquelle eingesetzt wird. Der Ausgang Charge Control Output (CCO) dient der Ladezustandskontrolle, d.h. der Überprüfung der zur Verfügung stehenden Versorgungsspannung. Der CCO-Anschluss führt einen High-Pegel $(1,8 \mathrm{~V})$, wenn VDD größer ist als $V \_O n$. Er führt einen Low-Pegel $(0 \mathrm{~V})$, wenn sich VDD zwischen $\mathrm{V} \_$on und $\mathrm{V} \_$off $(1,9 \mathrm{~V})$ befindet, und ist hochohmig (Tristate), wenn VDD

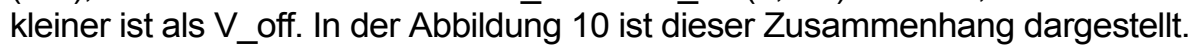

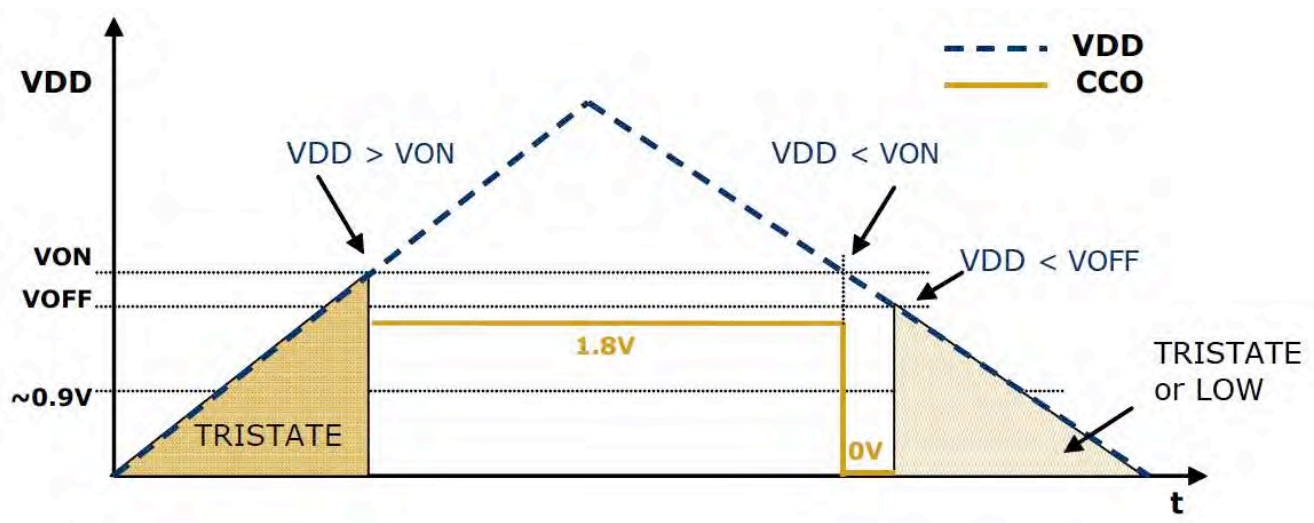

\section{Abbildung 10: Das Verhalten des CCO-Signals gibt Aufschluss über den aktuellen Ladezustand}

Der STM 300 unterstützt verschiedene Betriebsarten, wie u.a. Senden (14 mA), CPU/Mikrocontroller aktiv $(4 \mathrm{~mA})$, Standby $(1,3 \mathrm{~mA})$, Shortterm Sleep $(15 \mu \mathrm{A}$ oder Deep Sleep $(0,2 \mu \mathrm{A})$ mit dem geringsten Stromverbrauch. Das System befindet sich die meiste Zeit im Deep Sleep-Modus, der software-gesteuert, manuell per Taster oder automatisch per Aktivitätssensor verlassen wird, so dass daraufhin eine EKGMessung mit dem Senden der Daten stattfindet.

Zwischen den Telegrammen wird der Mikrocontroller für eine kurze Zeit in den Shortterm Sleep-Modus versetzt, bei dem der DVDD-Spannungsausgang abgeschaltet wird. Die Module sind von EnOcean für Einzelmessungen vorgesehen, deshalb ist eine kurze Sendepause bzw. eine Totzeit von einigen zehn Millisekunden notwendig, damit das Empfangsmodul genügend Verarbeitungszeit zur Verfügung hat.

\section{Energy Harvesting und Energy Management}

Da die Energieversorgung des EKG-Systems autark und wartungsfrei zu erfolgen hat, wird als Spannungsquelle eine Indoor-Solarzelle (AM1815) verwendet. Die Solarzelle der Firma Sanyo hat eine Größe von 56,1 mm x 45,2 mm und liefert bei einer relativ schwachen Beleuchtungsstärke von 200 Ix und einer Zellspannung von $3 \mathrm{~V}$ einen Strom von ca. $42 \mu \mathrm{A}$. Die Leerlaufspannung der Solarzelle liegt bei $4,9 \mathrm{~V}$ und der Kurzschlussstrom bei $47 \mu \mathrm{A}$. Weil die Beleuchtungsstärke in einem normal beleuchteten Büroraum ca. $600 \mathrm{Ix}$ beträgt, lässt sich eine realistische Abschätzung für den erwarteten Solarstrom machen, der im Bereich von ca. $140 \mu \mathrm{A}$ liegt.

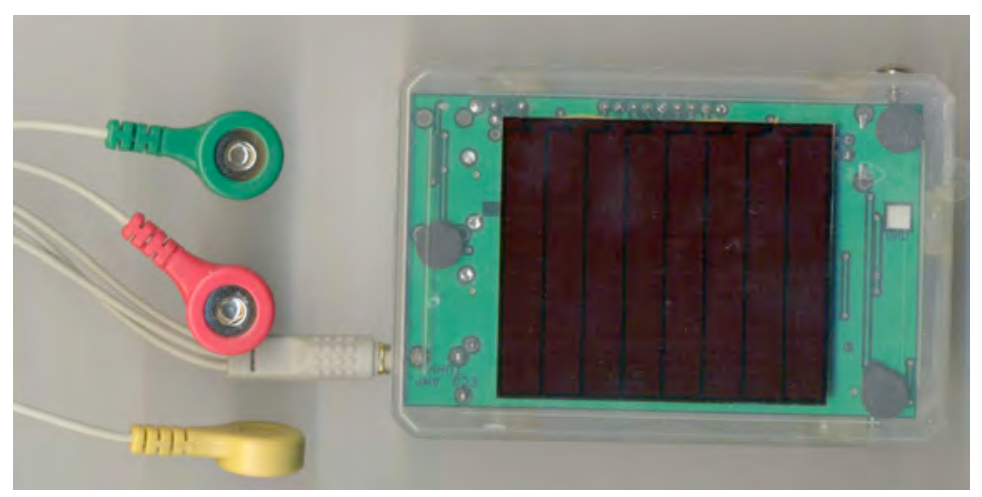

Abbildung 11: Montiertes System mit der Solarzelle.

Die EKG-Messschaltung soll für Langzeituntersuchungen eingesetzt werden, so dass eine permanente Messung und Messwertübertragung nicht notwendig und mit dieser Energiequelle allein auch nicht 
möglich ist. Das Funkmodul benötigt zum Senden einen (kurzzeitigen) Strom von ca. 20 mA, was einen entsprechenden Energiespeicher erfordert. Während eines Messzyklus wird das Messsystem dann aus diesem Speicher versorgt und nicht direkt von der Solarzelle. Als Ladungsspeicher sind die Doppelschichtkondensatoren geeignet, die oftmals als Gold Caps bezeichnet werden, obwohl dies eine Bezeichnung der Firma Panasonic ist und diese Kondensatortypen bei anderen Firmen deshalb auch unter Bezeichnungen wie Super Caps oder Supercapacitors geführt werden. Die Doppelschichtkondensatoren sind hinsichtlich der Ladungskapazität, des Innenwiderstandes und des Leckstromes mit Bedacht auszuwählen. Für das System ist ein Goldcap-Kondensator der Firma Panasonic mit einer Kapazität von 220 mF und einem Innenwiderstand von $\leq 75 \Omega$ vorgesehen.

Mit einer einfachen Berechnung ist überprüfbar, ob die Kapazität für diese Anwendung ausreichend ist. Der STM 300-Controller wird üblicherweise im Versorgungsspannungsbereich von 2,5 V bis 4,5 V betrieben. Das heißt, die Ladungsmenge $(Q)$, die aus dem Goldcap-Kondensator bezogen werden darf, beträgt:

$$
\Delta \mathrm{Q}=\Delta \mathrm{U} \cdot \mathrm{C}=\left(\mathrm{U}_{\mathrm{v}}-\mathrm{U}_{\mathrm{m}}\right) \cdot \mathrm{C}=(4,5 \mathrm{~V}-2,5 \mathrm{~V}) \cdot 220 \mathrm{mF}=440 \mathrm{mAs}
$$

Durch Messungen bei einer Systemmessdauer von 1,6 s und einer Abtastfrequenz von $120 \mathrm{~Hz}$ wurde für einen Messzyklus die notwenige Ladungsmenge von $52 \mathrm{mAs}$ ermittelt, was bedeutet, dass mit der Gesamtladungsmenge mindestens acht Messungen ausgeführt werden können. Somit erweist sich die Größe des Kondensators von 220 mF als optimal, weil größere Kapazitäten einen höheren Leckstrom verursachen und die Ladezeit verlängern würden.

Grundsätzlich verursacht der hohe Innenwiderstand eines Goldcap-Kondensators hohe Spannungseinbrüche bei hohen Strombelastungen. Im Sendemodus fließt kurzzeitig ein Strom von 20 $\mathrm{mA}$, der bei einem Innenwiderstand von $75 \Omega$ einem Spannungseinbruch von 1,5 V hervorruft. Wenn die Versorgungsspannung den Wert von 2,1 V unterschreitet, wird das STM300-Modul automatisch in den Schlafmodus versetzt und der Programmablauf unterbrochen. Um diesem Verhalten entgegenzuwirken, ist parallel zum Goldcap ein zusätzlicher Kondensator als Puffer angeschlossen. Hierfür eignen sich am besten Elektrolytkondensatoren, die bei großen Kapazitäten den niedrigsten Leckstrom aller üblichen Kondensatoren aufweisen. Der ausgewählte Elektrolytkondensator hat eine Kapazität von $470 \mu \mathrm{F}$ und einen Innenwiderstand von $900 \mathrm{~m} \Omega$. Aufgrund dieses verhältnismäßig kleinen Innenwiderstandes unterstützt der Elektrolytkondensator den Goldcap-Kondensator bei den hohen kurzzeitigen Belastungen und verhindert einen unzulässigen Spannungseinbruch der Versorgungsspannung.

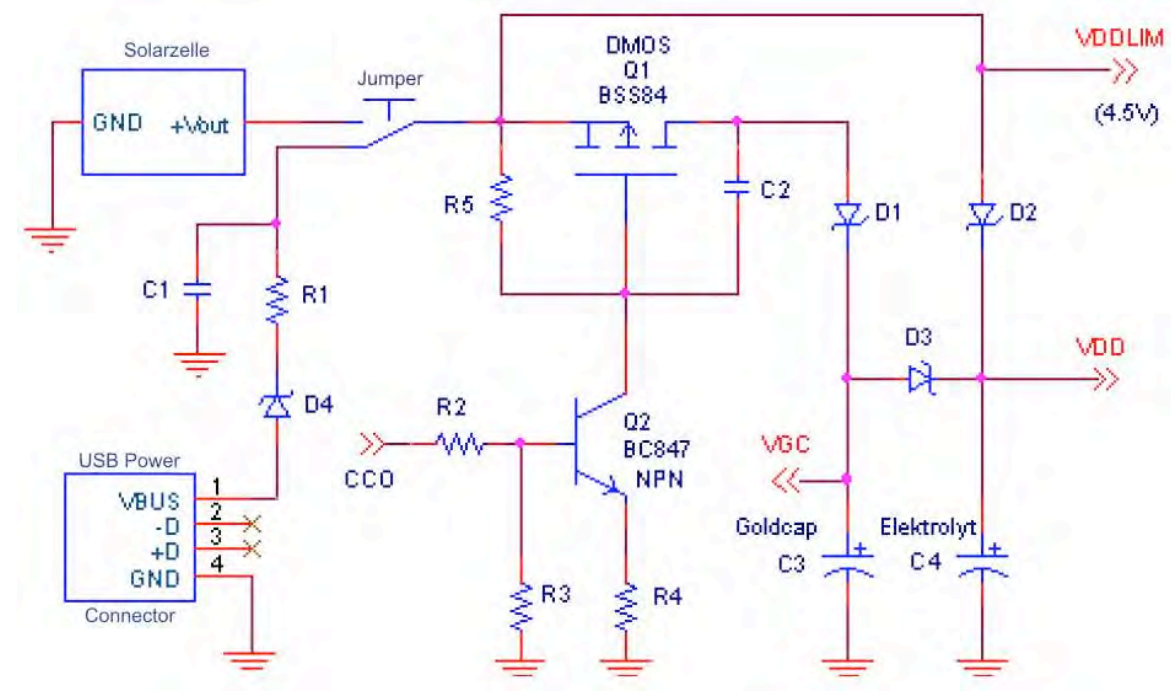

Abbildung 12: Schaltung des Energiemanagements

Die Spannung von der Solarzelle wird auf direktem Weg zum Elektrolytkondensator $\left(\mathrm{C}_{4}\right)$ und über einen p-Kanal-MOSFET (BSS84) zum Goldcap-Kondensator $\left(C_{3}\right)$ geführt. Der Jumper in der Schaltung (Abbildung 12) wird lediglich für den Entwicklungsprozess und den Testbetrieb umgesetzt, damit über den USB-Anschluss eine Versorgung der Schaltung erfolgen kann. Der Ladungstransfer zu den Speicherkondensatoren wird durch den NPN-Transistor (BC847) und mithilfe des STM 300-Moduls gesteuert. Zunächst wird der Elektrolytkondensator aufgeladen, und zwar so lange, bis die Begrenzungsspannung (VDDLIM), die vom STM300-Modul permanent überwacht wird, den Schwellwert von 4,5 V erreicht hat. 
Danach wird der Kontrollausgang (CCO) des STM300-Moduls aktiviert, der NPN-Transistor wird leitend und der Gate-Anschluss des DMOS-Transistors wird auf Massepotential (GND) gezogen. Damit entsteht eine Differenzspannung $\left(\mathrm{V}_{\mathrm{SG}}\right)$ zwischen Source und Gate, die den DMOS-Transistor aus dem hochohmigen Zustand in den niederohmigen Zustand versetzt. Der DMOS-Transistor ist dann leitend, und die Solarspannung wird zum Goldcap-Kondensator geführt.

Während der Goldcap-Kondensator aufgeladen wird, bleibt die Spannung $\left(\mathrm{V}_{\mathrm{DD}}\right)$ am Elektrolytkondensator konstant. Diese Spannung $\left(V_{D D}\right)$ wird als Versorgungsspannung für das Messsystem zur Verfügung gestellt und darf den Schwellwert von 4,5 V nicht überschreiten. Die Schottky-Dioden $\left(D_{1}, D_{2}\right)$, die mit den Speicherkondensatoren in Reihe geschaltet sind, lassen den Solarstrom nur in der definierten Flussrichtung fließen und sollen die Entladung der Kondensatoren verhindern, wenn kein Licht auf die Solarzelle fällt. Eine dritte Schottky-Diode $\left(D_{3}\right)$ wird zwischen die Speicherkondensatoren angeschlossen, so dass der Goldcap-Kondensator zum Elektrolyt-Kondensator parallel geschaltet ist.

In der Praxis hat es sich gezeigt, dass die Versorgungsspannung während eines Mess- oder Sendevorganges aufgrund eines zu niedrigen Energievorrates einbrechen kann, so dass vor einem Zyklus überprüft werden sollte, ob er ohne Fehler zu Ende geführt werden kann. Hierfür ist mindestens eine Kondensatorspannung (VGC) von $3 \mathrm{~V}$ notwendig, die mithilfe einer einfachen Transistorschaltung (Abbildung 13) durch das STM 300-Modul am Goldcap-Kondensator gemessen werden kann. Der Bipolartransistor (Q1) wird über den ADIO3-Pin des STM 300 eingeschaltet, kurz darauf die Spannung über den hochohmigen Spannungsteiler am Eingang ADIO1 des STM 300 gemessen und abschließend wird der Transistor sofort wieder abgeschaltet, so dass kein weiterer Strom verbraucht wird. Ist die Spannung ausreichend hoch, wird eine EKG-Messung ausgeführt, andernfalls wird der STM 300 wieder zurück in den Deep Sleep-Modus versetzt.

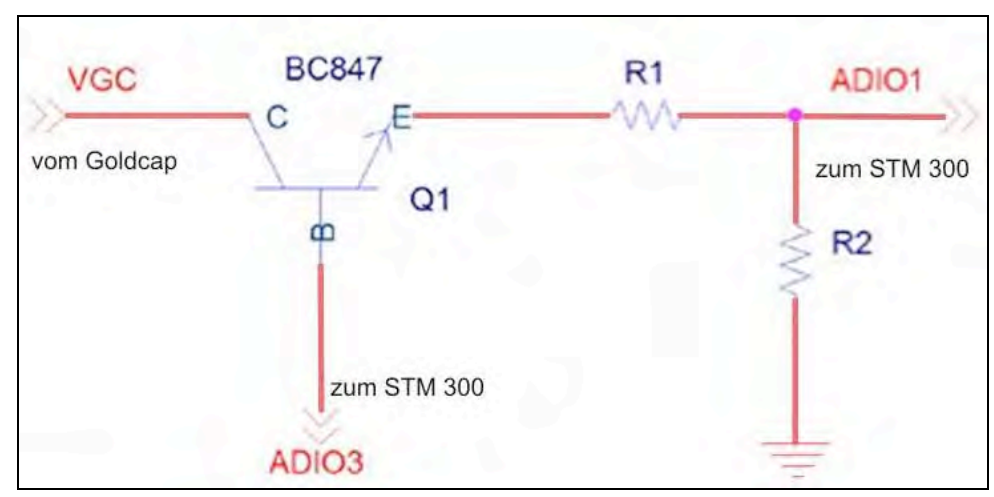

Abbildung 13: Ob genügend Energie für die Ausführung eines Zyklus zur Verfügung steht, wird durch eine einfache Transistorschaltung mit Spannungsteiler festgestellt.

\section{Aktivierungsmethoden}

Als Aufwachbedingungen aus dem Deep Sleep-Modus sind eine Timer-Funktion, die Betätigung eines Tasters sowie ein Aktivitätssensor implementiert, die nur bei Vorhandensein ausreichender Energie ausgelöst werden können, woraufhin eine EKG-Messung stattfindet. Die Timer-Funktion wird programmgesteuert und typischerweise alle zehn Minuten ausgeführt. Eine manuelle Auslösung ist mit dem Taster möglich, der den STM 300 über den WAKE0-Eingang aktiviert. Der Aktivitätssensor bildet eine Aufwachbedingung, die bei starken Lageänderungen des gesamten Systems (mithin des Probanden) ausgelöst wird. 

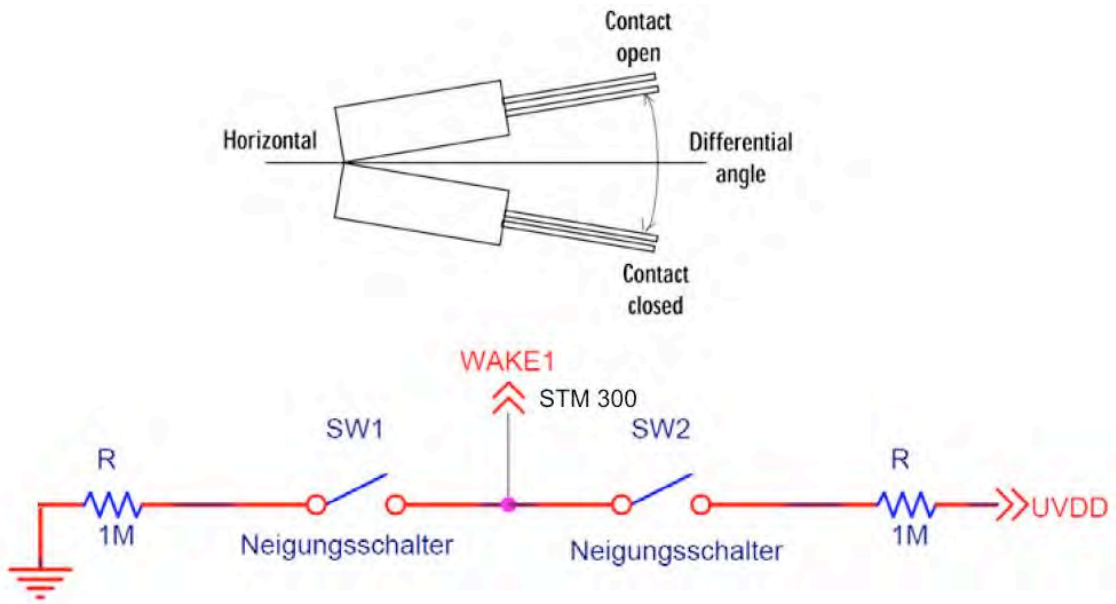

\section{Abbildung 14: Neigungsschalter als Aktivitätssensoren zur Auslösung einer EKG-Aufzeichnung}

Dazu werden zwei Neigungsschalter [5] entsprechend eines Wechselschalters eingesetzt. Diese bestehen aus einem Zylinder mit einer eingeschlossenen Kupferkugel und zwei Anschlüssen. Je nachdem wie sich der Zylinder neigt, verändert sich die Lage der Metallkugel. Dadurch sind die Kontakte einmal kurzgeschlossen und einmal offen. Die Funktion eines Wechselschalters wird dabei durch eine unterschiedliche Ausrichtung der beiden Neigungsschalter erreicht. Dabei schaltet der eine bei vertikaler und der andere bei horizontaler Lage. Dadurch ändert sich bei einer Bewegung des Systems auch der Pegel am WAKE1-Pin, was die Auslösung einer Messung zur Folge hat.

\section{Literatur:}

[1] K. Dembowski, Energy Harvesting für die Mikroelektronik: Energieeffiziente und -autarke Lösungen für drahtlose Sensorsysteme, ISBN 978-3-8007-3234-0, VDE Verlag GmbH, 2011

[2] B. Fuchs, Integrierte Sensorschaltungen zur EKG- und EEG-Ableitung mit prädiktiver Signalverarbeitung, Berichte aus der Elektrotechnik, Shaker Verlag Aachen, 2004

[3] Ambu GmbH, Datasheet Ambu Blue Sensor, http://www.ambu.de/patientmonitoring, 2011

[4] Enocean $\mathrm{GmbH}$, User Manuals and Data Sheets, http://www.enocean.com/de, 2011

[5] Comus Group of Companies, CM 1320 Product Data Sheet, 2003

[6] K. Hamann, EKG-Interpretation, http://www.ekg-interpretation.de/wordpress/?tag=einthoven, 2011 